Fecha de recepción: febrero 2019 Fecha de aceptación: julio 2019 Versión final: noviembre 2020

\section{Laboratorios de Transformación para un Futuro Sostenible}

Cristina Zurbriggen ${ }^{1}$, Mariana González Lago ${ }^{2}$, María Mancilla García ${ }^{3}$ y Sebastián Gatica ${ }^{4}$

Resumen: El presente artículo tiene por objetivo analizar los Laboratorios de transformación social con el fin de abordar problemas sociales complejos. Estos laboratorios se han convertido en espacios abiertos de diseño experimental que vincula diferentes actores (co-creación) y saberes (transdisciplinario y transectorial) a través de un proceso iterativo en la forma de abordar problemas complejos hacia un desarrollo sostenible. Por consiguiente, el presente paper busca reflexionar sobre los desafíos de cómo la actitud de diseño social y el proceso de diseño tiene que estar basada en clave de futuro y experimental así como la relevancia de desarrollar una cultura de la innovación y pensamiento resiliente (habilidades, competencia, actitudes y valores).

Palabras clave: innovación social, pensamiento resiliente, diseño en políticas públicas, laboratorios de transformación social, desarrollo sostenible.

[Resúmenes en inglés y portugués en la página 143]

(1) Instituto de Ciencia Política, Facultad de Ciencias Sociales, Universidad de la República, Instituto Sudamericano para Estudios sobre Resiliencia y Sostenibilidad, SARAS, Uruguay.

(2) Institute for Sustainable Futures, University of Technology, Sydney, Australia.

(3) Stockholm Resilience Centre, Stockholm University, Sweden.

(4) Universidad Católica de Chile- Director del CoLab.

\title{
Introducción
}

El gran desafío que enfrentan las sociedades actuales es cómo generar una reflexión crítica en la acción para co-construir un futuro socio-ambiental más sostenible. En los últimos tiempos el mundo se ha visto sometido a un proceso de transformación que se evidencia en sociedades cada vez turbulentas, más fluidas, más conectadas, menos sólidas, más desiguales, con una mayor proximidad a los límites planetarios. El cambio de época en que nos encontramos nos obliga a pensar y actuar de manera diferente. No solo necesitamos ser capaces de reaccionar a cambios que se producen, la clave está en cuán rápido vamos a ser capaces de cambiar, de abordar decididamente la complejidad de las problemáticas del siglo XXI para avanzar hacia un futuro socio-ambiental más sostenible y democrático. 
El interrogante que se presenta es cómo navegar en un paisaje incierto donde existe una multiplicidad de actores con intereses divergentes. Las urgencias que nos toca vivir tienen una característica transversal: tienden a ser el resultado de problemáticas interdependientes y complejas. Problemáticas que generalmente no pueden resolverse desde la perspectiva y el conocimiento de una sola disciplina o un solo actor (público, privado o de la sociedad civil), y por tanto, nos colocan en situaciones en las que debemos actuar sin contar necesariamente con un panorama completo del problema ni con toda la información que deseamos para guiarnos.

Este artículo tiene por objetivo presentar un nuevo modelo de laboratorios de innovación social, como el que se está desarrollado en el South American Institute for Resilience and Sustainability Studies (SARAS). Este modelo aspira a la convergencia de disciplinas y actores para trabajar en el encuadre colaborativo de problemas socio-ambientales y en la co-creación de soluciones adaptativas. Su finalidad es trabajar en la interfaz social, ecológica y política, alineándose con el abordaje emergente de laboratorios para la transformación o T-Labs. Con este cometido, el artículo se ha estructurado en tres partes. En la primera parte se presentan los principales aportes del pensamiento resiliente, el de la innovación social y enfoques emergentes en política pública como brújula para comenzar a identificar las capacidades necesarias para desarrollar una infraestructura más robusta para la transformación e innovación en escenarios complejos. En segundo lugar, inspirados en estos enfoques presentamos el modelo desarrollado en SARAS de Transformation Lab (T-Lab), orientado al trabajo con problemas socio-ambientales. Por último, reflexionamos sobre estrategias y desafíos para América Latina en materia de estos nuevos modelos de laboratorio para la transformación.

\section{Un marco conceptual para el T-Lab: los enfoques convergentes}

Alcanzar un planeta sostenible requiere repensar patrones de comportamiento social (Westley y Laban, 2015) para conceptualizar la articulación de los seres humanos con la naturaleza, como parte de sistemas socio-ecológicos, donde la gobernanza de los mismos esté basada en valores de solidaridad, reciprocidad y sostenibilidad (Dash, 2015).

Desde diferentes enfoques se ha señalado la necesidad de producir el conocimiento necesario y explorar los valores que pueden conducir a la transformación hacia sociedades sostenibles (Ahlqvist y Rhisiart, 2015). En este proceso en transición, el pensamiento de la resiliencia (Folke, 2016) y otros nuevos paradigmas como el de diseño de política pública (Peters, 2015, 2017) y el de innovación social (Manzini, 2015; Westley y Laban, 2015) nos aportan elementos para entender y actuar para el cambio social. Estos abordajes nos invitan a aprender a vivir con la incertidumbre; aumentar la diversidad de todos los componentes de un sistema (diversidad de actores involucrados, etc.), combinar diferentes tipos de conocimiento y aprendizaje; y crear oportunidades para la transformación.

El desafío consiste en generar una nueva meta-narrativa (Roe, 1994) para interpretar y actuar en este mundo complejo, alejándonos de las formas convencionales y dominantes de pensar y hacer. Por ello partimos de la hipótesis que para abordar los problemas del siglo XXI y articular el cambio social, se necesita un cambio cultural. 


\section{El Pensamiento de Resiliencia}

La literatura sobre la resiliencia de los sistemas socio-ecológicos (SES) comprende estos sistemas como adaptativos y complejos, esto implica concentrarse en su capacidad de renovación, reorganización y desarrollo, donde las perturbaciones se consideran parte de la dinámica del sistema y representan oportunidades de cambio (Gunderson y Holling, 2002; Walker et al., 2004; Walker y Salt, 2006).

Desde este enfoque se reconoce que los cambios en los sistemas ecológicos tendrán un impacto en los sistemas sociales y viceversa, que son intrincados y no lineales, caracterizados por ciclos de retroalimentación e incertidumbre (Berkes et al., 2003). Folke (2016) define la resiliencia como la capacidad de las personas, comunidades, sociedades, y culturas de vivir y desarrollarse en ambientes con cambios constantes (esperados e inesperados).

El "pensamiento de resiliencia" establece tres principios fundamentales:

- Los problemas ambientales no se pueden analizar ni comprender separados de su contexto social.

- La incertidumbre y la sorpresa son atributos de los SES y debemos aprender a vivir con ellos.

- El cambio es intrínsecamente complejo; por lo tanto, cuestiones como el cambio global no pueden abordarse en un solo nivel de organización, sino considerando la vinculación de los diferentes niveles.

El pensamiento de resiliencia va más allá de una formulación estrecha de la resiliencia como capacidad de resistir las conmociones. Examina tanto la adaptabilidad como la capacidad de transformación de los sistemas socio-ecológicos, en tanto propiedades que influyen en la resiliencia a diferentes escalas de gobernanza (desde la comunidad local hasta la nacional, regional y mundial) (Folke, 2016).

Es por tanto un concepto dinámico, preocupado en cómo navegar la complejidad y la incertidumbre a través de esos niveles y escalas (Berkes et al., 2003; Cash et al., 2006; Cumming et al., 2013) en un planeta dominado por humanos (Steffen et al. 2007) como agentes de cambio (Folke, 2016).

De esta manera, el concepto de resiliencia permite hacer la distinción entre la capacidad de mantener un sistema en su estado actual (adaptabilidad) y la capacidad de crear deliberadamente un sistema nuevo y más deseable (transformabilidad) (véase Walker et al., 2004). Esta distinción puede ayudar a identificar partes de un sistema socioecológico particular donde la transformación es preferible al status quo (o resiliencia en el sentido estricto).

Para ello se hace relevante comprender la dinámica de cambio, es decir, es necesario entender la estructura y los patrones de las interacciones intra e inter subsistemas para explorar e incrementar su resiliencia y la capacidad de transformación. La capacidad de transformación se define como la capacidad de crear un sistema fundamentalmente nuevo, cuando las condiciones ecológicas, económicas o sociales (incluidas las políticas) hacen que el sistema existente sea insostenible (Walker et al., 2004), lo que conlleva crear puntos de entrada diversos al sistema, nuevas formas de hacer las cosas y, a menudo, cambios en las escalas de retroalimentaciones cruciales (Chapin et al., 2009). 
En este proceso, la adaptación y la transformación requieren la gestión de información nueva o cambiante, y por tanto, el manejo de múltiples tipos de conocimiento. Las estrategias prácticas en relación con el aumento de la resiliencia de los SES son: (1) aprender a vivir con el cambio y la incertidumbre; (2) aumentar la diversidad de todos los componentes de los SES (diversidad de objetivos económicos, diversidad de recursos naturales utilizados, diversidad de actores involucrados, etc.); (3) combinar diferentes tipos de conocimiento y aprendizaje; y (4) crear oportunidades para la autoorganización y los vínculos entre los diferentes niveles (por ejemplo, entre las esferas nacional y local) (Berkes et. al., 2003).

La anticipación, a pesar de que el conocimiento sea imperfecto, el aprender a lidiar con las no linealidades y los diversos umbrales de incertidumbre y resistencia, son elementos claves para navegar un SES. Por ello, los modelos de gobernanza deben generar procesos más colaborativos e inclusivos dentro de sistemas institucionales policéntricos, evitando la fragmentación y manteniéndose flexibles, adaptables, experimentales, con una capacidad real para responder al cambio, así como la capacidad de aprender a diseñar e implementar estrategias para superar las condiciones adversas actuales y futuras (Chapin et al., 2009).

\section{Pensamiento de Resiliencia e Innovación Social}

Dentro del pensamiento resiliente, se ha desarrollado una línea teórica sobre el papel de la innovación social (por ejemplo, Biggs et al., 2010; Westley y Antadze, 2010; Westley et al., 2013) y la agencia estratégica (Westley et al., 2013) en los procesos de transformación. En este caso, las trayectorias del cambio transformador se consideran emergentes de la interacción entre las condiciones institucionales top-down y las innovaciones bottom-up (catalítica y disruptiva), apalancadas a través de agencias institucionales y redes en múltiples niveles de organización como pueden propiciar los laboratorios de innovación social. Como sostiene Dash (2017), la esencia del SES es relacionar a los humanos y la naturaleza en una ontología superior de la agencia humana enraizada en el cambio.

En particular, una fuente de inspiración clave en esta línea ha sido el trabajo de Frances Westley (2008) que vincula el enfoque del pensamiento de diseño con el enfoque sistémico de la teoría de la resiliencia. Westley introdujo el modelo del llamado "ciclo adaptativo" (Gunderson y Holling 2002) para conceptualizar la innovación como un ciclo infinito, que representa la no linealidad de la innovación social y está formado por cuatro fases (Westley, 2008) que permiten la comprensión de la innovación incremental y radical en un SES.

Asimismo, Manzini (2015) aborda la relación entre el enfoque de diseño, el cambio social y cultura resiliente, definiendo el diseño social como "todo lo que el diseño experto puede hacer para activar, mantener y orientar los procesos de cambio social hacia la sostenibilidad" (Manzini, 2015, p. 62l). El diseño es política, es decir, el diseño social debe estar orientado a generar bienes y servicios hacia un desarrollo sostenible.

Es así que plantea que la gran transformación es un proceso de cambio en el que la humanidad está empezando a entender los límites del planeta y que también nos está llevando a hacer un mejor uso de la conectividad disponible. A partir de allí es posible esbozar un 
escenario de diseño construido sobre una cultura que une lo local con lo global (localismo cosmopolita) y sobre una infraestructura resiliente capaz de recalificar el trabajo y acercar la producción al consumo (sistema distribuido) (Manzini, 2015, p. 2).

\section{El enfoque de diseño en políticas públicas}

Dentro de los paradigmas emergentes en los estudios de política pública que están reflexionando sobre la complejidad de los problemas públicos, podemos mencionar el enfoque diseño (Peters, 2015, 2017) y los aportes del experimentalismo (Ansell y Bartenberger and 2016). Peters (2015) afirma que para innovar en políticas públicas y abordar la complejidad de los problemas, primero tenemos que conceptualizar la política pública como prospectiva y experimental a diferencia de la producción de conocimiento en ciencias sociales que es explicativa y retrospectiva. Con este fin, propone retornar al enfoque de diseño (policy design) desarrollado en los años 80 (como ejemplos, Dryzek 1983; Bobrow y Dryzek 1987; Schon y Rein 1994; Dryzek and Ripley 1984; Linder y Peters 1984, 1991) y que se abandonó en los 90 con el avance del paradigma de la Nueva Gerencia Pública.

Como señala Peters (2017), el diseño tiene menos que ver con los métodos y mucho más con la cultura del diseño (habilidades, competencias, actitudes, valores). Esto se refleja mejor por la diferencia entre la "actitud de diseño" y la "actitud de decisión" (esta última se centra en la mayoría de los enfoques tecnocráticos de la política). La "actitud de decisión" está representada de forma dominante en el pensamiento de gestión y el análisis de políticas positivista que se centran en elegir entre alternativas, mientras que supone que éstas son fáciles de generar. En términos prácticos, los responsables de la toma de decisiones se presentan con un conjunto limitado de opciones y la intención es buscar medios elaborados para elegir entre ellos. Sin embargo, la "actitud de diseño" consiste en identificar nuevas alternativas que podrían conducir a la mejor posible dadas las habilidades, el tiempo y los recursos disponibles, es decir, una alternativa "satisfactoria" (Peters, 2017).

El verdadero "problema de diseño" no es "resolver" un problema, sino aproximar la solución idealizada (la "desiderata" original) a la "real" que surge al final del proceso de diseño. Por lo tanto, el diseño no resuelve los problemas y no debe considerarse un proceso de establecimiento de metas o cumplimiento de metas. El foco principal está en la llamada "desiderata" (Nelson y Stolterman, 2014), que se considera una intención o un objetivo de diseño. En este marco, el éxito se evalúa no mediante el logro de un objetivo determinado, sino por el grado en que el resultado final se alinea con la intención original. Sin embargo, el diseño no es solo un proceso "salvaje" y caótico, siempre hay un cierto andamiaje, pero esta estructura generalmente pretende ser una "brújula”. La dificultad para diseñar, por lo tanto, es decidir qué tan extenso puede ser el andamiaje y cuán extensa debe ser la consideración de las alternativas.

El énfasis está en la interacción y la deliberación entre distintos actores con el fin de permitir la innovación. El aprendizaje en la arena de la política consiste en aprender haciendo, en la generación de significados, la creación de la identidad y el fortalecimiento de la participación. En otras palabras, el aprendizaje reflexivo y cooperativo es clave para mejorar y entrenar la capacidad colectiva de dar sentido (making-sense y sense-making) a la novedad, 
a lo no-conocido, a lo emergente para que sea inteligible, interpretable y efectivo para orientarnos en la toma de decisiones en los términos del cambio.

Por otra parte, para reconocer fenómenos emergentes se requiere anticipación, no solo para generar conocimiento en relación al futuro (horizonte temporal), sino también para el fortalecimiento de capacidades y competencias -individuales y colectivas- anticipatorias que orientan (dan y crean) sentido a lo nuevo, desconocido en el presente. La anticipación es clave (como una acción, como una competencia y como un fenómeno sistémico emergente) para los procesos de cambio social que implican disrupciones tales como las que caracterizan estos tiempos, siendo una parte intrínseca del aprendizaje y la resiliencia creativa. Esto enfatiza la reflexión crítica que constituye la columna vertebral del aprendizaje (Argyris y Schön, 1978, 1996) y la determinación de "ventanas" para resolver preguntas emergentes permite a los investigadores y participantes desarrollar y probar teorías e ideas a través de la acción y facilitar el aprendizaje sobre situaciones complejas.

\section{Experimentalismo y políticas públicas}

El diseño de política no solo requiere anticipación sino también un alto nivel de experimentación, como un proceso en el que la colaboración intersectorial promueve la reflexión crítica sobre estrategias para abordar desafíos complejos y sistémicos (Ansell y Bartenberger, 2016; Ansell y Geyer 2016).

Tanto el enfoque de diseño como el experimental plantean una serie de aspectos comunes. En primer lugar, señalan que el diseño de políticas públicas se ha tecnocratizado y se ha olvidado de la política. Por ello, exigen ser consciente de la manera en que los distintos marcos de análisis de los actores (framing), definen y discuten los problemas, y cómo éstos chocan, convergen y cambian. Estos marcos de análisis traducidos en discurso se relacionan con lo que priorizamos y en los instrumentos de políticas que elegimos. Por tanto, la fundamentación de la política no se puede basar solo en la evidencia científica, sino que se basa en valores que están implícitos en el propio discurso y accionar (Schon y Rein, 1994). Una segunda característica es la centralidad del entendimiento sistémico del problema y las causas profundas del mismo, rechazando el enfoque cartesiano de racionalidad instrumental. La política es una construcción social más que científica. Una tercera característica común es que el enmarque de una política siempre ocurre en un contexto anidado, es decir, cuando se modifica alguna dimensión del contexto (interno, externo, global) puede ser que ya la política no funcione. El diseño de políticas se ve como la dialéctica entre construcción y "adaptación ecológica" entre "principio» y «contexto».

Un cuarto elemento en común es que señalan al cambio, a un nuevo orden de las cosas, como el objetivo del diseño de políticas, y este cambio ocurre en un mundo lleno de incertidumbres y, por lo tanto, está destinado a ser desordenado y difuso, con muchas consecuencias. Una quinta esencialidad del diseño de políticas, como actividad, es que se anida en un proceso social, o un sistema llamado multi-actor. El sexto elemento es que siempre requiere conocimiento sobre situaciones pasadas, presentes y futuras. Por lo tanto, la finalidad del diseño en política es realizar un cambio intencionado en un mundo social ayudado por algunos conocimientos pero también con una gran incertidumbre. 
El proceso experimental está altamente relacionado con una cultura de la "complejidad pragmática” (Ansell y Geyer, 2016) en la cual la reflexión y deliberación permiten reconocer la naturaleza compleja e incierta del problema, abarcando el potencial de razonabilidad. La cultura de la complejidad pragmática va más allá de la visión positivista (racionalidad, reduccionismo, predictibilidad, determinismo). Es una investigación abierta que concibe el diseño de la política como un proceso colaborativo de resolución de problemas basado en la deliberación, la experimentación, el aprendizaje y la especificidad del contexto, en el que los actores son cuestionados y replanteados conjuntamente con sus valores y comprensión, donde el poder y la visiones conflictivas son consideradas como relevantes. Un experimento generativo aborda un problema particular enraizado en la experiencia y la situación de las personas que realizan el experimento (vivencial y orientada a problemas). No hay un a priori, una sensación de certeza de que ésta sea la única o la correcta solución al problema, sino que se aprende al tratar de abordar el problema (learning by doing); la solución se refina continuamente a medida que se implementa (iterativa) y, simultáneamente, se construye la capacidad de la implementación transformativa (Ansell y Bartenberger, 2016). Las actualizaciones iterativas asociadas con los experimentos generativos pueden reflejar la negociación constante para avanzar hacia una solución que satisfaga a los diferentes interesados. Es poco probable que un experimento generativo avance sin un cierto grado de acuerdo compartido del problema en sí mismo y la conveniencia de aprender sobre él. Bos, Brown y Farrely (2013) sostienen que una agenda de aprendizaje compartida es un punto de partida esencial para un experimento de política pública.

El gran desafío es cómo llevar comenzar hacer dialogar el pensamiento de resiliencia y el pensamiento de diseño sistémico (no tecnocrático) como una nueva metanarrativa que nos aparte de patrón positivista habitual de toma de decisiones. En los procesos de transformación necesitamos generar espacios para la experimentación que faciliten la innovación y la transformación creativa.

\section{El modelo T-LAB}

Un T-Lab es un espacio para fortalecer los Homo Reciprocans y Sociolugus (Dash, 2015), ya que lo que se necesita es un "cambio de piel" (Leff, 2009, p. 105). Este tipo de actores integraría valores de solidaridad, reciprocidad y sostenibilidad como medio para alcanzar un bienestar moral y ecológico.

Tversky and Kahneman (1974) demostraron que en contextos reales de incertidumbre y complejidad, los comportamientos humanos no están determinados por el principio de maximización de la utilidad. Ambas naturalezas, la egoísta (Homo Economicus), y la altruista (Homo Reciprocans), se encuentran en la naturaleza humana. Es la estructura social que construye y fomenta uno u otro comportamiento. En la misma dirección Polanyi (1957) ya afirmó que la institucionalización de la economía capitalista, de acuerdo con la construcción social considerada como universal del Homo Economicus, condujo a la mercantilización de la vida y la destrucción de las bases humanas y naturales de la sociedad. La concepción hegemónica del ser humano como criatura egoísta implica la destrucción de la naturaleza y la promoción de la desigualdad social. Pero Polanyi reconoció 
que es posible desarrollar una relación más compleja con la sociedad y la economía, con nuevas instituciones para guiar el comportamiento humano de una manera que mantenga la vida de todos los miembros de la sociedad.

De hecho, el modelo de los T-Lab desarrolla y alienta a los actores reflexivos sobre los nuevos valores colectivos. Un T-Lab fomenta un proceso de aprendizaje creativo donde las partes interesadas pueden explorar la colaboración transdisciplinaria para abordar problemas socioecológicos complejos y transformar ideas nuevas en acciones prácticas, basadas en la deliberación, la experimentación y la especificidad del contexto, y en las que los actores cuestionan y replantean conjuntamente sus valores (Dewey, 1927; Schön, 1983).

En este contexto, los T- Lab tienen un nuevo rol fundamental a jugar en la agenda de sostenibilidad, con una investigación-acción para la transformación y con un paradigma de compromiso social.

\section{El modelo T-Lab de SARAS}

El T-Lab de SARAS busca promover una cultura reflexiva que experimente en torno a la integración de Ciencia, Arte y Política a fin de construir capacidades para la transformación. Es por eso que en este espacio se convoca a científicos naturales y sociales, artistas, otros actores relevantes y tomadores de decisiones para enmarcar los desafíos socioecológicos y trabajar con una perspectiva crítica de sistemas, conectando humanos y naturaleza. Esto implica una nueva epistemología política de la intervención social/pública basada en el pensamiento resiliente, de innovación social, de enfoque de diseño y experimentalismo, que nos permite tratar problemas complejos con una perspectiva sistémica y generar transformaciones sociales (Dewey, 1927, 1933; Schön, 1983; Ansell y Geyer 2016).

El T-LAB es un espacio para la colaboración transdisciplinaria donde se produce la creación colectiva de conocimiento a partir de: estar juntos (diferentes partes interesadas), utilizar juntos diferentes tipos de conocimiento (talentos, habilidades) y hacer juntos (desarrollo, investigación, innovación, co-creación de nuevas ideas, nuevas políticas, representación/visualización, comunicación) y actuar para enfrentar desafíos relevantes y desarrollar una agenda de aprendizaje compartido.

El modelo se sustenta en los siguientes pilares:

- Visión sistémica: las ideas e iniciativas en el T-Lab aspiran a ser sistémicas por naturaleza, basada en principios socio-ambientales. Dar sentido a las relaciones entre las diferentes entidades asociadas a una situación compleja (en comprensión). La intención principal no es obtener un conocimiento exhaustivo completo de las situaciones, sino más bien adquirir una mejor apreciación de las dinámicas más amplias y profundas con el fin de mejorar la situación. Ir más allá de tratar con una parte del todo o los síntomas y abordar la raíz de las cosas que no están funcionando (experimentación generativa) para dar lugar al surgimiento de iniciativas provisionales y adaptativas que reflejen el carácter experimental del proceso. Se trata de generar pensamiento sistémico de una situación y parcialidad entre diferentes partes interesadas. La intención aquí es perturbar, perturbar y, por lo tanto, provocar un nuevo pensamiento sistémico. 
- Inclusión social: para que las personas con diversas perspectivas trabajen de forma colectiva. La intención principal aquí no es abarcar todas las perspectivas sobre un problema predeterminado para resolverlo, sino más bien para permitir que surjan posibilidades en la remodelación de una situación problemática para mejorar su resolución.

- Pensamiento crítico: explorar y reconciliar (con responsabilidad y reflexión crítica) cuestiones éticas y relaciones de poder, ambas expresiones de problemas fronterizos asociados con entendimientos parciales inevitables del pensamiento crítico (Midgley, 2000).

\section{Conectando ciencia, arte y política}

El T-Lab busca conectar ciencia, arte y política, estimulando el balance del pensamiento dual (Scheffer et al., 2015), asociando los procesos analíticos de razonamiento con el pensamiento intuitivo y experimental para abordar la dinámica total del sistema (ver Tabla 1 a continuación) (la "consiliencia”). Como señala Scheffer (2015, p. 3), "Recordando la definición de creatividad como la generación de nuevas ideas útiles, los mejores resultados se obtienen mediante un tango íntimo entre los dos sistemas" (creatividad y razonamiento).

\begin{tabular}{|c|c|}
\hline Análisis & Síntesis \\
\hline (Separación) & (Unificación) \\
\hline Racional & Emocional \\
\hline Lógico & Intuitivo/pragmatico \\
\hline Deductivo & Inductivo \\
\hline Soluciones & Paradigmas, platformas \\
\hline Pensar a través de... & Pensar haciendo \\
\hline Una disciplina & Multiples disciplinas, forma T \\
\hline Causalidad & Impacto, valor, difusión \\
\hline
\end{tabular}

Tabla 1. La "consiliencia”. Fuente: Bason (2010). 
El pensamiento intuitivo conduce a un proceso de prueba y error en el que los actores pueden no darse cuenta de todas las razones que los llevan a probar una solución u otra (Snow, 1959; Scheffer et al., 2015; Glatzeder, 2011). Además, el pensamiento intuitivo incluye: empatía, es decir, una observación profunda de las necesidades y emociones de los demás; imaginación, particularmente una búsqueda optimista de soluciones; y visualización, lo que permite facilitar lo difícil a través de la producción colectiva de imágenes.

En este proceso, las artes tienen el potencial de ayudar a fomentar formas intuitivas, experienciales y menos inhibidas de explorar y representar dinámicas de sistemas y posiciones de las personas en estas dinámicas (Scheffer et al., 2015).

Los enfoques artísticos son parte integral de la concepción del T-Lab y esto por tres razones fundamentales. En primer lugar, los enfoques artísticos ayudan a hacer explícitos e incorporar los aspectos emocionales de la gobernanza ambiental (Curtis, 2009; Curtis et al., 2012; Wiek e Iwaniec, 2014; Scheffer et al., 2015). En segundo lugar, los enfoques artísticos ayudan a visualizar escenarios imaginarios e inventar los pasos que podrían conducirles. En tercer lugar, los enfoques artísticos sirven como puentes para hacer explícitas y fortalecer las conexiones existentes entre las personas, y entre las personas y los elementos naturales (Inwood, 2008, Kagan, 2008, Selman et al., 2010).

Estos tres elementos juntos fomentan diferentes enfoques de aprendizaje de manera altamente exploratoria y motivadora (McNaughton, 2004; Flowers et al., 2015; Scheffer et al, 2015). Quizás aún más importante, permiten participar en una discusión significativa sobre los valores que guían el proceso de gobernanza y los corroboran en términos de cuidado. De hecho, el T-Lab asume completamente que el conocimiento no es de valor neutral y aborda explícitamente la cuestión de los valores. El pensamiento analítico viene a complementar la exploración artística de los valores haciéndolos explícitos y los escenarios que benefician a los actores (Ansell y Geyer, 2016).

Entre estos desafíos está la búsqueda de métodos novedosos que puedan respaldar los procesos de aprendizaje transformacional y el empoderamiento de las personas que conducen a la transformación social.

En el T-Lab, se fomenta:

- La "consiliencia" entre el conocimiento, los valores y las perspectivas en el diálogo con las partes interesadas, vinculando diversas fuentes de conocimiento científico con experiencias personales, emociones y juicios éticos

- Comunicación y traducción de complejidad,

- Reflexión social, deliberación y comprensión pública,

- Construcción de identidades socio-ecológicas y conciencia ecológica,

- Compromiso y compromiso emocional que lleva a la acción (Heras y Tabara, 2013).

Por lo tanto, el conocimiento, el aprendizaje y el cambio social se entrelazan en formas muy complejas y no evidentes. El arte nos ayuda a incorporar la perspectiva de múltiples partes interesadas, comprendiendo su valor, el uso del sistema, los arreglos institucionales que crean formal e informalmente (gobernanza), identidades subjetivas, cultura y relación de poder, que son necesarios para los procesos de cambio y para crear capacidades de resiliencia socioecológica. 


\section{Los desafíos en la construcción de los T- LABs}

En la construcción de los Lab es importante identificar el tipo de capacidades que debemos desarrollar para fortalecerlos. En este sentido, identificamos los siguientes desafíos: El primer desafío es generar capacidad de anticipación para gestionar la incertidumbre, con el fin movilizar a las personas y transformar las visiones construidas colectivamente en acción. La incertidumbre en la formulación de políticas radica no solo en la imprevisibilidad de los sistemas naturales, sino también en el conocimiento imperfecto sobre el comportamiento humano, así como en la variabilidad e imprevisibilidad inherente de dicho comportamiento. Por ejemplo, más investigaciones e innovaciones tecnológicas ayudarían a reducir la incertidumbre epistémica, mientras que poco se puede hacer para reducir la incertidumbre ontológica. El hecho de que haya múltiples partes interesadas involucradas en el proceso de políticas, cada una con sus propios sistemas de creencias, puntos de vista, preferencias e intereses, y por lo tanto sus propias interpretaciones de la misma información, da lugar a un nuevo tipo de incertidumbre: la ambigüedad, una situación en la cual un tomador de decisiones no tiene una comprensión única y completa (Brugnach et al., 2008). Para sortear estas dificultades el pensamiento anticipatorio puede ayudar al diálogo reflexivo desde una perspectiva intelectual y emocional, incluyendo el descubrimiento de los diferentes marcos interpretativos (framings), cosmovisiones subyacentes a nuestro sistema de valores que determinan nuestra acción.

El segundo desafío es generar capacidad de síntesis del conocimiento de una manera transdisciplinaria para comprender el problema. Estas prácticas implican una interacción continua entre actores de diferentes subsistemas sociales (investigación, política, sociedad civil, sector privado), para vincular diferentes perspectivas, tipos de conocimiento (científicos y experienciales) con el fin de alcanzar una comprensión más profunda del problema en la vida real y generar una brújula para una mejor toma de decisión (Bason, 2010; Ostrom, 1990).

El proceso de aprendizaje implica la exploración e integración de conocimiento útil, ya sea tácito o codificado. La base conceptual más relevante para la transdisciplinariedad es la visión sistémica del problema como proceso de construcción social y aprendizaje en acción (Hirsch et al., 2010) como dos actos inseparables y simultáneos (Westberg y Polk, 2016). Se rechaza la noción de que el conocimiento puede ser neutral en cuanto a los valores, por tanto, ello implica, relacionar e interconectar hechos, juicios, visiones, valores, intereses, epistemologías, escalas de tiempo, escalas geográficas y visiones del mundo (Bammer, 2013) no exentas de conflictos.

El tercer desafío es generar la capacidad de experimentar para desarrollar espacios tangibles en la contexto actual para permitir el cambio. La experimentación requiere el desarrollo y el uso de una gama de herramientas experimentales que van más allá de los ensayos controlados aleatorios (Ansell y Bartenberger, 2016).

El cuarto desafío es innovar en la forma de evaluar y monitorear los procesos de innovación pública más allá de los modelos convencionales basados a menudo en relaciones de causa y efecto. Un gran aporte ha sido el de Patton cuya formulación de la "evaluación de desarrollo" se constituirá en un nuevo paradigma de evaluación orientado al aprendizaje, la innovación y adaptación en sistemas dinámicos y complejos. 
De acuerdo a Patton, es necesario incorporar enfoques reflexivos en la evaluación de procesos donde existen diversos actores involucrados y que como resultado de esas interacciones múltiples, no está claro cómo o si la intervención conducirá a un resultado específico. Reconociendo la imprevisibilidad inherente de cualquier camino de cambio tomado, se supone que se requiere de una gestión integrada y adaptativa, de sondeo y aprendizaje, y una reflexión recurrente sobre los patrones emergentes (Patton, 2011; Snowden y Boone, 2007). Es por ello, que Patton habla de la necesidad de intervenciones y gerenciamiento adaptativo, ya que no se trata de "testear" un modelo sino de generarlo constantemente. Las técnicas tradicionales de evaluación no sirven para el cambio sistémico y hacer frente a lo inesperado e impredecible. Estas se basan en modelos probados a través de la evidencia y las buenas prácticas, y que se supone pueden ser escalables. La evaluación de desarrollo no ofrece un modelo prescripto, sino principios de cambio que son adaptables a distintos contextos y que se identifican en el gerenciamiento adaptativo de abajo hacia arriba.

\section{Conclusiones}

Entender cómo se puede construir y apoyar la capacidad de transformación requiere intervenir en los puntos profundos de apalancamiento, incluidos los procesos de toma de decisiones, desarrollo de políticas, cultura, valores e intereses (Meadows, 1999).

La gobernanza para navegar el cambio requiere un doble enfoque: en adaptación (es decir, respuestas y estrategias a corto y largo plazo para amortiguar perturbaciones y proporcionar capacidad para lidiar con el cambio y la incertidumbre), y en transformación (es decir, estrategias para crear un nuevo sistema fundamentalmente cuando las condiciones actuales hacen que el actual se mantenga).

Esta nueva ola de la innovación tiene mucho más que ver con un cambio cultural, con construir nuevas narrativas de innovación pública resiliente. Y estas transformaciones tienen mucho menos que ver con la planificación y control, sino más sobre la creación de nuevas capacidades en los gobiernos para navegar en los procesos de cambio.

La innovación pública, como un proceso de colaboración en el abordaje de problemas públicos, basado en la anticipación, la deliberación, la experimentación, el aprendizaje y la especificidad del contexto, en la que los actores cuestionan y replantean conjuntamente sus valores y su comprensión, implica una nueva epistemología política de la intervención pública, basada en una cultura de pragmatismo experimental que nos permite tratar problemas complejos con una perspectiva sistémica y generar transformaciones sociales.

\section{Bibliografía}

Acevedo, S. e Dassen, N. (2016). Innovando para una mejor gestión: la contribución de los laboratorios de innovación pública. Washington: BID.

Ahlqvist, T. e Rhisiart, M. (2015). Emerging pathways for critical futures research: changing contexts and impacts of social theory. Futures 71, 91-104. 
Antadze, N. \& Westley, F. R. (2012). Impact metrics for social innovation: barriers or bridges to radical change?. Journal of Social Entrepreneurship, 3(2), 133-15

Ansell, C. K. and Bartenberger M. (2016). Varieties of experimentalism Ecological Economics 130 (2016) 64-73.

Ansell, C. \& Geyer, R. (2016). 'Pragmatic complexity'a new foundation for moving beyond 'evidence-based policy making'?. Policy Studies, 38(2), 149-167.

Ansell, Ch. \& Torfing, J. (2014). Public Innovation through Collaboration and Design, Routledge Critical Studies in Public Management.

Argyris, C. \& Schön, D. (1978). Organizational learning: A theory of action perspective. Addison Wesley, Reading, Mass.

Bammer, G. (2013). Disciplining interdisciplinarity: Integration and implementation sci-ences for researching complex real-world problems. Canberra: ANU Press

Bason, C. (2010). Leading Public Sector Innovation: Co-creating for a Better Society. Bristol: Policy Press.

Berkes, F.; Colding, J. and Folke, C. ( 2003). Navigating social-ecological systems: building resilience for complexity and change. Cambridge: Cambridge University Press..

Bobrow, D. B. and Dryzek, J. S. (1987). Policy Analysis by Design. Pittsburgh: University of Pittsburgh Press.

Bos, J. J.; Brown, R. R. \& Farrelly, M. A. (2013). A design framework for creating social learning situations. Global Environmental Change, 23(2), 398-412.

Bovaird, T. and Loeffler, E. ( 2012). "From Engagement to Co-Production: The Contribution of Users and Communities to Outcomes and Public Value." Voluntas 23 (4): 1119-1138.

Boyle, D. (2010). Local economics and co-production. Development, 53(3), 319-324.

Brugnach, M.; Dewulf, A.; Pahl-Wostl, C. and Taillieu, T. (2008). Toward a relational concept of uncertainty: about knowing too little, knowing too differently, and accepting not to know. Ecology and Society 13(2):30.

Cash, D. W.; Adger, W.; Berkes, F.; Garden, P.; Lebel, L.; Olsson, P.; Pritchard, L. and Young, O. (2006). Scale and cross-scale dynamics: governance and information in a multilevel world. Ecology and Society 11(2):8.

Chesbrough, H. (2003). Open Innovation - The new imperative for Creating and Profiting from Technology.

Chapin III, F. et. al. (2009). Ecosystem stewardship: sustainability strategies for a rapidly changing planet. Trends Ecol. Evol. 25 (4), 241-249.

Cumming, G. S.; Olsson, P.; Chapin III, F. S. and Holling, C. S. (2013). Resilience, experimentation, and scale mismatches in social ecological landscapes. Landscape Ecology 28:1139-1150.

Dash, A. (2015). An Epistemological Reflection on Social and Solidarity Economy, Forum for Social Economics, 45:1, 61-87.

Dewey, J. (1927). The Public and Its Problems. New York: Holt.

Drieschova, A. \& Fischhendler, I. (2012). A toolkit of mechanisms to reduce uncertainty in international water treaties. Jerusalem: The Hebrew University of Jerusalem. CLICO project.

Dryzek, J. S. (1983). Don't Toss Coins into Garbage Cans: A Prologue to Policy Design, Journal of Public Policy 3, 345-67. 
Feola, G. (2015). Societal transformation in response to global environmental change: a review of emerging concepts. Ambio 44:376-390.

Fischer, J.; Gardner, T. A.; Bennett, E. M.; Balvanera, P.; Biggs, R.; Carpenter, S.; Daw, T.; Folke, C.; Hill, R. and Hughes, T. P. (2015). Advancing sustainability through mainstreaming a social - ecological systems perspective.

Folke, C. (2016). Resilience (Republished). Ecology and Society 21(4):44.

Folke, C.; Carpenter, S.; Elmqvist, T.; Gunderson, L.; Holling, C. S. and Walker, B. (2002). Resilience and sustainable development:building adaptive capacity in a world of transformations. Ambio 31:437-440.

Gamble, J.A. (2008). A developmental evaluation primer. Montreal: JW McConnell Family Foundation.

Geels, F. W. and Kemp, R. ( 2006). Transitions, transformations, and reproduction: dynamics in socio-technical systems. Pages 227-257 in M. D. McKelvey and M. Holmén, editors. Flexibility and stability in the innovating economy. Oxford Scholarship Online Monographs, Oxford, UK.

Gibson, R. B.; Kay, B.; Loorbach, D.; McCormick, K.; Parodi, O.; Rauschmayer, F.; Schneidewind, U.; Stauffacher, M.; Stelzer, F.; Trencher, G.; Venjakob, J.; Vergragt, P. J.; von Wehrden, H. \& Westley, F. R. (2016). Learning through evaluation -a tentative evaluative scheme for sustainability transition experiments, J. Clean. Prod. 169 (2017) 61-76.

Giddens, A. (1976). New Rules of Sociological Method: A Positive Critique of Interpretative Sociologies. London: Hutchinson.

Glatzeder, B. (2011). “Two modes of thinking: evidence from crosscultural psychology” en Han, S. y Pöppel, E. (ed). Culture and neural frames of cognition and communication: on thinking. Springer: Berlin Heidelberg.

Gunderson, L. H.; Holling, C. S. y Light, S. S. (1995). Barriers and Bridges to the Renewal of Ecosystems and Institutions. New York: Columbia University Press.

Gunderson, L. H. \& Holling, C. S. (ed) (2002). Panarchy. Washington: Island Press.

Hirsch, G. H.; Pohl C. \& Bammer, G. (2010). «Chapter 30: Solving problems through transdisciplinary research", en Froderman, R. et. al. (eds.). The Oxford Handbook of Interdisciplinarity, Oxford, oup, pp. 431-452.

Holling, C. S. and Chambers, A. D. (1973). Resource science: the nurture of an infant. BioScience 23:13-20. http://dx.doi. org/10.2307/1296362

Holling, C. S. and Meffe, G. K. (1996). Command and control and the pathology of natural resource management. Conservation Biology 10:328-337. http://dx.doi.org/10.1046 /j.1523-1739.1996.10020328.

Inwood, H. J. (2008). At a crossroads: situating place-based art education. Canadian Journal of Environmental Education 13 (1):29-41.

Jones, J. C. (1992). Design Methods: seeds of human futures. Hoboken: John Wiley and Sons. Jones, P. (2014). Systemic Design Principles for Complex Social Systems, In Social Systems and Design, Gary Metcalf (ed). Volume 1 of the Translational Systems Science Series, Springer Verlag.

Kagan, S. (2008). Sustainability: a new frontier for the arts and cultures. Frankfurt: Vas Verlag Fur Akademisch. 
Leach, M. et. al. (2012). Transforming innovation for sustainability. Ecology and Society 17(2):11.

Leeuw, S. (2011). Tipping toward sustainability: emerging pathways of transformation. Ambio 40 (7), 762-780.

Leff, E. (2009). Degrowth, or deconstruction of the economy: Towards a sustainable world. Occasional Paper Series No.6 In Contours of Climate Justice, Critical Currents. Uppsala: Dag Hammarskjo"ld Foundation.

Luederitz, C.; Abson, D. J.; Audet, R. and Lang, D. J. (2016). Many pathways toward sustainability: not conflict but co-learning between transition narratives, Sustainability Science.

Linder, S. H. and Peters, B. G. (1984). From Social Theory to Policy Design, Journal of Public Policy 4, 237-59.

Linder, S. H. and Peters, B. G. (1991). The Logic of Public Policy Design, Knowledge \& Policy 4, 125-51.

Loorbach, D. (2007). Transition management: new mode of governance for sustainable development. Utrecht: International Books.

Manzini, E. (2015). Design, When Everybody Designs. Cambridge: The MIT Press.

Manzini, E. y Staszowski, E. (Eds.). (2013). Public and Collaborative. Exploring the Intersection of Design, Social Innovation and Public Policy. Estados Unidos: Desis Network.

Meadows, D. H. (1999). Leverage points: places to intervene in a system. The Sustainability Institute.

Meadows, D. et. al. (1972). The Limits to Growth: A Report for the Club of Rome's Project on the Predicament of Mankind. New York: Universe Books.

Miller, T. R.; Wiek, A.; Sarewitz, D.; Robinson, J.; Olsson, L.; Kriebel, D. and Loorbach, D. (2014). The future of sustainability science: A solutions-oriented research agenda. Sustainability Science 9: 239-246.

Mulgan, G. et.al. (2007). Social Innovation: What Is, Why It Matters And How It Can Be Accelerated. Oxford: Said Business School.

Nesta (2016). Designing for Public Services. London: Nesta. Disponible en: http://www.nesta. org.uk/sites/default/files/nesta_ideo_guide_021216.pdf

Nesta (2014). I-teams: The teams and funds making innovation happen in governments around the world. London: Nesta with Bloomberg Philanthropies.

O'Brien, K. (2012). Global environmental change II: from adaptation to deliberate transformation. Progress in Human Geography 36:667-676. http://dx.doi.org/10. $1177 / 0309132511425767$.

OCDE (2017). Fostering Innovation in the public service, OCDE. Disponible en http://www. oecd.org/gov/fostering-innovation-in-the-public-sector-9789264270879-en.htm

OCDE (2014). https://www.oecd.org/innovating-the-public-sector/Background-report.pdf

Olsson, P. et. al. (2014). Sustainability transformations: a resilience perspective. Ecology and Society 19(4):1.

Olsson, P.; Gunderson, L.H.; Carpenter, S. R.; Ryan, P.; Lebel, L.; Folke, C. \& Holling, C.S. (2006). Shooting the rapids: navigating transitions to adaptive governance of socialecological systems. Ecol. Soc. 11 (1), 18.

Ostrom, E. (1990). Governing the commons: the evolution of institutions for collective action. New York: Cambridge University Press. 
Patton, M. Q. (2011). Essentials of utilization-focused evaluation. Sage Publications.

Pelling, M.; O'Brien, K. \& Matyas, D. (2014). Adaptation and transformation. Clim. Chang. 2014, 133, 113-127.

Pestoff, V. (2009), “Towards a Paradigm of Democratic Participation: Citizen Participation and Co-Production of Personal Social Services in Sweden." Annals of Public and Cooperative Economics 80 (2): 197-224.

Peters, G. (2015). "Public Policy: a design perspective" en Advanced Introduction to Public Policy, Elgar, pp. 1-12.

Peters, G. (2017). Policy Design: From Technocracy to Complexity, and Beyond University, Toronto, manuscrito.

Polanyi, K. (1957). The Great Transformation, Beacon Press, Boston, Massachusetts, EE.UU.

Rein, M. \& Schön, D. (1993). Reframing Policy Discourse en Fischer, Frank, and John Forester, en The Argumentative Turn in Policy Analysis and Planning. Durham, NC: Duke University Press. Pag 145-167.

Roe, E. (1994). Narrative policy analysis theory and practice. Durham: Duke University Press.

Selman, P. et. al. (2010). Reconnecting with a neglected river through imaginative engagement. Ecology and Society 15(3):18.

Schön, D. (1978). "Generative metaphor: A perspective on problem setting in social policy," en Ortony, A. Metaphor and Thoughts. Cambridge: Cambridge, pp. 264-272.

Schön, D. (1984). The Reflective Practitioner: How professionals think in action. New York: Basic Books

Schon, D. \& Rein, M. (1994). Frame Reflection: Toward the Resolution of Intractable Policy Controversies. New York: Basic Books.

Snowden, D. et. al. (2007). A leader's framework for decision making. Harvard business review, 85(11), 68 .

Steffen, W.; Crutzen, J. and McNeill, J. R. (2007). The Anthropocene: Are humans now overwhelming the great forces of nature? Ambio 36:614-621.

Scheffer, M.; Bascompte, J.; Bjordam, T. K.; Carpenter, S. R.; Clarke, L. B.; Folke, C.; Marquet, P.; Mazzeo, N.; Meerhoff, M.; Sala, O. and Westley, F. R. (2015). Dual thinking for scientists. Ecology and Society 20(2):3.

Snow, C. P. 1998 (1959). The Two Cultures. Cambridge \& New York: Cambridge University Press.

Tschakert, P. \& Dietrich, K. A. ( 2010). Aprendizaje anticipado para la adaptación al cambio climático y la resiliencia. Ecology and Society 15 (2): 11.

Tàbara, J. D. and Chabay, I. ( 2013). Coupling human information and knowledge systems with social-ecological systems change: reframing research, education, and policy for sustainability. Environmental Science and Policy28:71-81.

Tversky, A. \& Kahneman, D. (1974). Judgment under uncertainty: Heuristics and biases. science, 185(4157), 1124-1131.

Westberg, L. and Polk, M. (2016). The role of learning in transdisciplinary research: Moving from a normative concept to an analytical tool through a practice based approach. Sustainability Science, 11:385-397.

Walker, B.; Holling, C. S.; Carpenter, S. R. y Kinzig, A. (2004). Resilience, adaptability and transformability in social-ecological systems. Ecology and Society, 9 (2). 
Westley, F. y Laban, S. (2015). Social Innovation Lab Guide, Waterloo.

Westley, F.; Tjörnbo, O.; Schultz, L.; Olsson, P.; Folke, C.; Crona, B. and Bodin, Ö. (2013). A theory of transformative agency in linked social-ecological systems. Ecology and Society 18(3):27.

Westley, F. (2008). The Social Innovation Dynamic. Waterloo, ON: Social Innovation Generation/SiG@Waterloo.

Westley, F. y Zimmerman, P. (2006). Getting to maybe: how the world is changed. Toronto: Random House.

Ziervogel, G.; Cowen, A. \& Ziniades, J. (2016). Moving from Adaptive to Transformative Capacity: building Foundations for Inclusive, Thriving, and Regenerative Urban Settlements; Sustainability, 8,955.

Resumo: O objetivo deste artigo é analisar os laboratórios de transformação social para abordar problemas sociais complexos. Estes laboratórios tornaram-se espaços abertos de desenho experimental que ligam diferentes atores (co-criação) e conhecimento (transdisciplinar e transetorial) através de um processo iterativo na forma de abordar problemas complexos em direção ao desenvolvimento sustentável. Portanto, este trabalho procura refletir sobre os desafios de como a atitude do design social e do processo de design deve ser baseada na chave para o futuro e experimental, bem como a relevância de desenvolver uma cultura de inovação e pensamento resiliente (habilidades, competências, atitudes e valores).

Palavras chave: inovação social - pensamento resiliente - desenho em políticas públicas laboratórios de transformação social - desenvolvimento sustentável.

Abstract: The objective of this article is to analyze the Laboratories of social transformation in order to address complex social problems. These laboratories have become open spaces of experimental design that link different actors (co-creation) and knowledge (transdisciplinary and transectoral) through an iterative process in the way of addressing complex problems towards sustainable development. Therefore, this paper seeks to reflect on the challenges of how the attitude of social design and the design process has to be based on the key to the future and experimental, as well as the relevance of developing a culture of innovation and resilient thinking (skills, competence, attitudes and values).

Keywords: social innovation - resilient thinking - design in public policies - laboratories of social transformation - sustainable development. 